\title{
Competing interactions at the interface between ferromagnetic oxides revealed by spin-polarized neutron reflectometry
}

\author{
J.-H. Kim, ${ }^{1}$ I. Vrejoiu, ${ }^{2}$ Y. Khaydukov, ${ }^{1,5}$ T. Keller, ${ }^{1,5}$ J. Stahn, ${ }^{3}$ A. Rühm, ${ }^{4,5}$ D. K. Satapathy, ${ }^{6}$ V. Hinkov, ${ }^{1,7, *}$ and B. Keimer ${ }^{1, \dagger}$ \\ ${ }^{1}$ Max-Planck-Institut für Festköperforschung, D-70569 Stuttgart, Germany \\ ${ }^{2}$ Max-Planck-Institut für Mikrostrukturphysik, D-06120 Halle, Germany \\ ${ }^{3}$ Paul Scherrer Institut, Laboratory for Neutron Scattering, 5232 Villigen PSI, Switzerland \\ ${ }^{4}$ Max-Planck-Institut für Intelligente Systeme, D-70569 Stuttgart, Germany \\ ${ }^{5}$ Forschungsneutronenquelle Heinz Maier-Leibnitz (FRM-II), D-85748 Garching, Germany \\ ${ }^{6}$ University of Fribourg, Department of Physics and Fribourg Centre for Nanomaterials, CH-1700 Fribourg, Switzerland \\ ${ }^{7}$ Quantum Matter Institute, University of British Columbia, 2355 East Mall, Vancouver, British Columbia, Canada V6T 1Z4
}

(Received 21 November 2011; revised manuscript received 26 July 2012; published 8 November 2012)

\begin{abstract}
We have investigated the magnetization profiles in superlattices composed of the two ferromagnets $\mathrm{La}_{0.7} \mathrm{Sr}_{0.3} \mathrm{MnO}_{3}$ and $\mathrm{SrRuO}_{3}$ using spin-polarized neutron reflectometry. In combination with magnetometry, the neutron data indicate a noncollinear spin configuration where orientation of the Ru moments changes from in plane at the interface to out of plane deep inside the $\mathrm{SrRuO}_{3}$ layers. The spin structure originates in a competition between antiferromagnetic exchange interactions of $\mathrm{Mn}$ and $\mathrm{Ru}$ moments across the interface, and the magnetocrystalline anisotropy of the Ru moments, and it is closely related to the "exchange spring" structures previously observed in multilayers composed of ferromagnetic elements and alloys.
\end{abstract}

DOI: 10.1103/PhysRevB.86.180402

PACS number(s): 75.70.Cn, 75.47.Lx, 75.25.-j

The magnetotransport properties of metallic ferromagnetic oxides such as $\mathrm{La}_{0.7}(\mathrm{Sr}, \mathrm{Ca})_{0.3} \mathrm{MnO}_{3}$ and $\mathrm{SrRuO}_{3}$ have been the subject of a large-scale research effort. ${ }^{1-5}$ Recent advances in the synthesis of oxide-based heterostructures have now opened up perspectives for the controlled manipulation of the magnetic properties of these compounds, and for the transfer of ferromagnetic spin polarization into other materials. Ferromagnetic oxides have thus been incorporated in heterostructures with a large variety of materials including high-temperature superconductors, ${ }^{6-8}$ multiferroics,,${ }^{9,10}$ and carbon nanotubes. ${ }^{11}$ The electronic properties of such heterostructures are determined by the complex interplay between a multitude of parameters including the charge-carrier density, spin and orbital polarization, lattice distortions, and disorder, all of which are in general different from those in the constituent bulk materials. Since these parameters are difficult to probe in an interface-specific manner, current research efforts aimed at a quantitative description of the interfacial electronic properties require simple model systems in which the influence of individual parameters can be determined separately.

Recent experiments have identified epitaxial superlattices (SLs) composed of the two ferromagnets $\mathrm{La}_{0.7} \mathrm{Sr}_{0.3} \mathrm{MnO}_{3}$ (LSMO; bulk Curie temperature $T_{C}^{\mathrm{LSMO}}=364 \mathrm{~K}$, Ref. 12) and $\mathrm{SrRuO}_{3}\left(\mathrm{SRO}\right.$; bulk $T_{C}^{\mathrm{SRO}}=163 \mathrm{~K}$, Ref. 13) as interesting model systems in which the influence of magnetic interactions is dominant. ${ }^{14,15}$ Both materials share the same pseudocubic lattice structure, and they are metallic and orbitally degenerate at all temperatures. One therefore does not expect modulations of the charge-carrier density or interfacial orbital or lattice polarization to play major roles. Magnetometric measurements on LSMO/SRO SLs with 2-nm-thick LSMO and 5-nm-thick SRO layers (henceforth SL2/5) revealed an isotropically reduced net magnetization for $T<T_{C}^{\mathrm{SRO}}$, suggesting antiferromagnetic (AFM) interlayer exchange coupling (Fig. 1). The out-of-plane magnetization at low temperatures increases with increasing thickness of the SRO layers. Data on SL2/8 samples showed a suppressed magnetization for magnetic fields applied in the SL plane only, whereas the magnetization in a perpendicular field increases upon cooling below $T_{C}^{\mathrm{SRO}}$. The thickness-dependent magnetic properties have been interpreted as evidence of competing magnetic interactions at the interface. $^{14-16}$

Motivated by these results, we have carried out a specular polarized neutron reflectivity (PNR) study of the magnetization profiles in SL2/5 and SL2/8, comprising 15 bilayer repetitions, which were synthesized under identical conditions as those in Refs. 14 and 15. The uniform magnetization of our samples was found to be consistent with those of Refs. 14 and 15. The structural properties of the SLs were probed by $\mathrm{x}$-ray reflectometry with radiation of wavelength $1.54 \AA$. Figure 2 shows the reflected $\mathrm{x}$-ray intensity as a function of momentum transfer $q_{z}$ perpendicular to the SL plane, along with the results of least-squares fits performed with the GenX code. ${ }^{17}$ Since previously reported electron microscopy data showed that the interfaces between LSMO and SRO are affected by $\mathrm{Mn}-\mathrm{Ru}$ intermixing over 1-3 unit cells across the interface, ${ }^{18-20}$ we used two different structure models: model 1 without and model 2 with intermixing layers between the LSMO and SRO layers. The fitting parameters were each layer's thickness, interface roughness, and scattering length density (SLD). The SLDs of the LSMO and SRO layers were constrained to be within $10 \%$ from the bulk values, while the intermixing layer's value was free to vary. The fitting parameters for the inner 14 bilayers of LSMO and SRO were constrained to be identical, while those of the LSMO layer adjacent to the substrate and of the SRO surface layer were allowed to vary independently. The figure of merit (FOM) function is defined by $\frac{1}{N-1} \sum\left|\log \left(I_{\text {obs }}\right)-\log \left(I_{\text {calc }}\right)\right|$, where $N$ is the number of points and $I_{\mathrm{obs}}\left(I_{\mathrm{calc}}\right)$ is the experimental (calculated) reflected intensity. The layer thicknesses obtained from the best fits (where each FOM value is less than 0.1) 


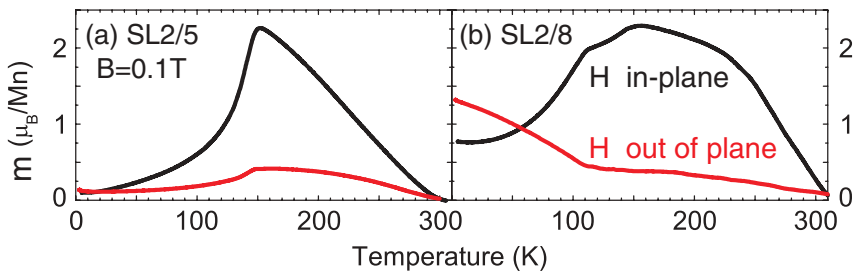

FIG. 1. (Color online) Magnetization curves of superlattices (a) SL2/5 and (b) SL2/8 with $0.1 \mathrm{~T}$.

are listed in Table I. The roughnesses of the topmost layers of SL2/5 and SL2/8 are 4.6 and $1.9 \AA$, respectively.

Even though model 1 generates correct Bragg peak positions [Figs. 2(a) and 2(b)], it fails to reproduce their intensities, in particular, for the higher orders which are highly sensitive to the LSMO/SRO interface. For instance, the fifth-order Bragg peak should be suppressed if the thickness ratio of the LSMO and SRO layers is 1:4. The disagreement can be resolved by including an intermixing layer, as realized in model 2 [Figs. 2(c) and 2(d)]. The thickness of the intermixing layers is about $6 \AA$, and the obtained SLD of the intermixing layers is the average value of the SLDs of LSMO and SRO (bottom rows in Figs. 3, 4 and 5). This is consistent with the electron microscopy data of Ref. 18, which were taken on small sections of similar specimens. Our results confirm that intermixing is present at interfaces throughout the SL sample.

The PNR measurements were performed at the time-offlight neutron reflectometer AMOR at SINQ (Paul Scherrer Institute, Villigen, Switzerland) and the angle-dispersive neutron reflectometer NREX at FRM-II (Garching, Germany). At AMOR the incidence angles were $0.5^{\circ}, 1^{\circ}, 2^{\circ}$, and $4^{\circ}$. At NREX the neutron wavelength was set to $\lambda=4.32 \AA$ and both polarizer and analyzer were used for observing non-spin-flip channels $\left(R^{++}\right.$and $R^{--}$, which originate from the nuclear structure and the in-plane magnetic components parallel to the
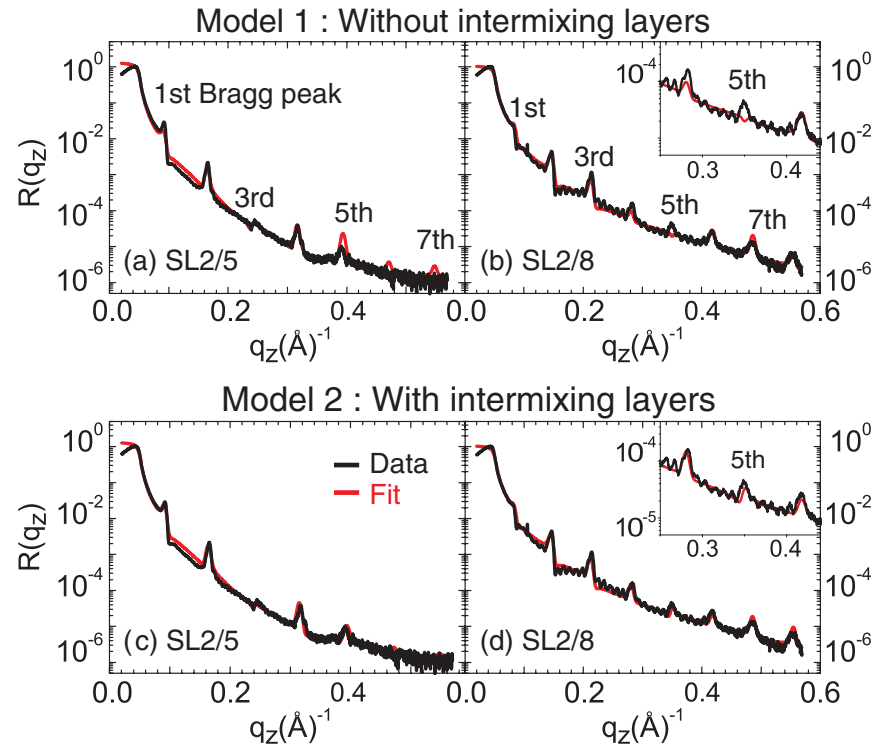

FIG. 2. (Color online) Hard x-ray reflectivity data of SL2/5 [(a) and (c)] and SL2/8 [(b) and (d)]. The insets in (b) and (d) show a magnified view around the fifth SL Bragg peak.
TABLE I. Layer thicknesses obtained from the fits. Errors are given in parentheses. The errors are calculated as a $5 \%$ increase in the optimal FOM, which is described in the text.

\begin{tabular}{lccccc}
\hline \hline & \multicolumn{2}{c}{ Model 1 } & & \multicolumn{2}{c}{ Model 2 } \\
\cline { 2 - 3 } \cline { 6 - 7 } \cline { 6 - 7 } & (a) SL2/5 & (b) SL2/8 & & (c) SL2/5 & (d) SL2/8 \\
\hline Top SRO & $47.1(7)$ & $74.4(4)$ & & $46.8(8)$ & $68.0(9)$ \\
LSMO & $24.7(4)$ & $20.6(2)$ & & $21.7(4)$ & $18.9(3)$ \\
Intermixing & & & & $5.9(1)$ & $5.5(2)$ \\
$\quad$ SRO & $55.8(5)$ & $72.2(2)$ & & $47.2(3)$ & $62.8(6)$ \\
Bottom LSMO & $25.5(5)$ & $25.5(5)$ & & $24.8(5)$ & $21.8(3)$ \\
\hline \hline
\end{tabular}

applied field) and spin-flip signals $\left(R^{+-}\right.$and $R^{-+}$, which arise from the in-plane magnetization perpendicular to the field). For both measurements, the samples were mounted in closed-cycle refrigerators, and the external magnetic field $H$ was applied perpendicular to the scattering plane and parallel to the film surface [sketch in Fig. 6(f)]. The measurements were carried out in $H=0.1 \mathrm{~T}$ after field cooling in the same magnetic field.
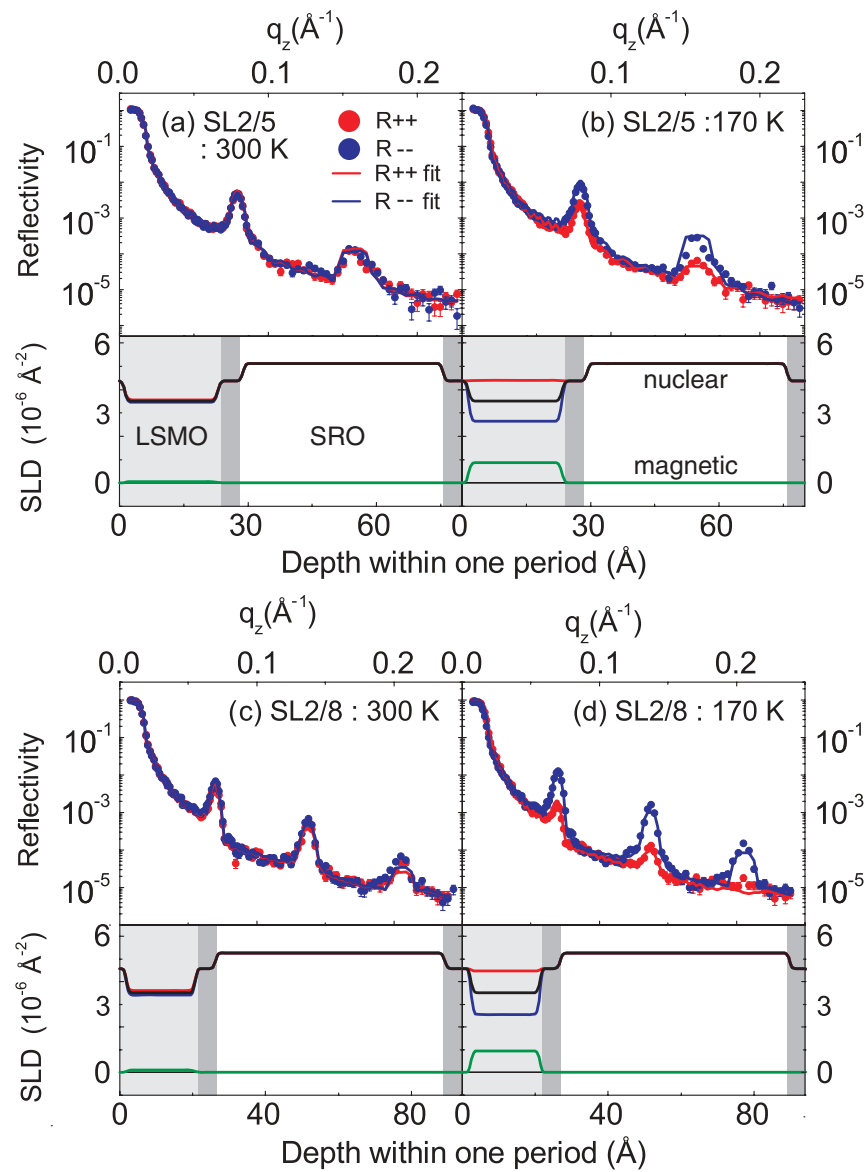

FIG. 3. (Color online) Polarized neutron reflectivity results on SL2/5 and SL2/8 at $300 \mathrm{~K}$ [(a) and (c)] and $170 \mathrm{~K}$ [(b) and (d)]. Red and blue points indicate the non-spin-flip $R^{++}$and $R^{--}$reflectivities, respectively. The experimental data are presented by symbols, while the lines are the results of fits described in the text. The structural and magnetic depth profiles of one bilayer corresponding to each temperature are shown below each fit. Dark gray regions indicate the intermixing layers. 


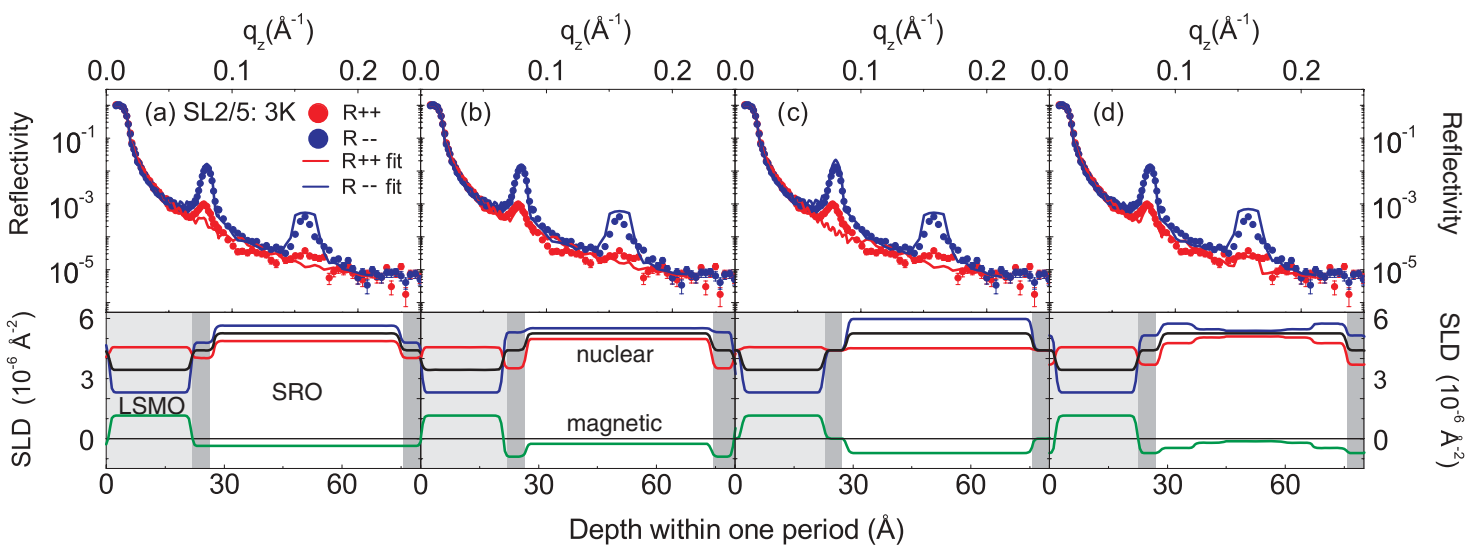

FIG. 4. (Color online) PNR spectra of SL2/5 at $3 \mathrm{~K}$. Each panel shows the fitting result obtained from the magnetic depth profile of one bilayer shown below each fit, which is described in the text. The symbols and colors were used in the same way as in Fig. 3 .

Figures 3(a) and 3(b) show PNR spectra of SL2/5 at 300 and $170 \mathrm{~K}$. At $300 \mathrm{~K}$, the Bragg reflections in the $R^{++}$ and $R^{--}$profiles have comparable intensities. At $T=170 \mathrm{~K}$ $\left(>T_{C}^{\mathrm{SRO}}\right)$, a clear difference between the intensities of the $R^{++}$and $R^{--}$reflections at the first and second SL Bragg peaks is observed. We carried out fits, keeping the structural parameters previously obtained by $\mathrm{x}$-ray reflectivity fixed, with the magnetic SLD of LSMO being the only free parameter [Figs. 3(a) and 3(b)]. The values of the moments were restricted not to exceed $4 \mu_{B}$ per Mn ion. In addition, the total magnetic moment obtained from all fits was constrained to be consistent with the magnetometric data at the respective temperature in Fig. $1\left(0.09 \mu_{B} / \mathrm{Mn}\right.$ at $300 \mathrm{~K}$ and $2.18 \mu_{B} / \mathrm{Mn}$ at $\left.170 \mathrm{~K}\right)$. The results indicate that at $300 \mathrm{~K}$, the $\mathrm{FM}$ ordered in-plane moment is small, consistent with $T_{C}^{\mathrm{LSMO}} \approx 300 \mathrm{~K}$ (somewhat reduced from the bulk value) determined by magnetometry. The moment increases upon cooling, inducing a clear difference between the $R^{++}$and $R^{--}$signals at $170 \mathrm{~K}$ where SRO is still paramagnetic. . $^{14,15}$

At $3 \mathrm{~K}<T_{C}^{\mathrm{SRO}}$ where the magnetic moments in the SRO layers couple antiferromagnetically to the ones in LSMO, there are even bigger differences between the $R^{++}$and $R^{--}$ signals in a wide range of $q_{z}$ (Fig. 4). For the fit of these data, the free parameters of the magnetic SLDs of the SRO and intermixing layers were additionally included, and the in-plane FM moment amplitude of SRO was restricted not to exceed $2 \mu_{B}$ per Ru ion. Also, the total magnetic moment was constrained to be consistent with $0.06 \mu_{B}$ per Mn ion obtained from the magnetometric data at $3 \mathrm{~K}$. As a starting point, we have constrained the magnetic profiles of SRO and intermixing layers to be homogeneous. As shown in Fig. 4(a), models incorporating this constraint provide poor fits especially for the first Bragg peak. To improve the fits, we allowed the magnetization of the SRO and of the intermixing layers to vary. The enhanced magnetization at the LSMO/SRO interface provides a well matched fit [Fig. 4(b)], while a magnetic model with a suppressed magnetization near the interfaces [Fig. 4(c)] and a sizable in-plane magnetization deep inside the SRO layer failed to yield satisfactory agreement with the data. To understand the enhanced magnetization at the interface and to further refine our fit, we used a model with a varied magnetization within the SRO layer. The SRO layers were divided into nine sublayers whose thicknesses and magnetic SLDs were constrained to be symmetric with respect to the center of a SRO layer. As shown in Fig. 4(d), with increasing distance from an intermixing layer in this magnetic model, the magnitude of the in-plane magnetization is reduced to almost zero within a range of $\sim 15 \AA$ around the center of the SRO layers. Compared to Fig. 4(b), this model provides an improved fit in the vicinity of the second-order peak. The

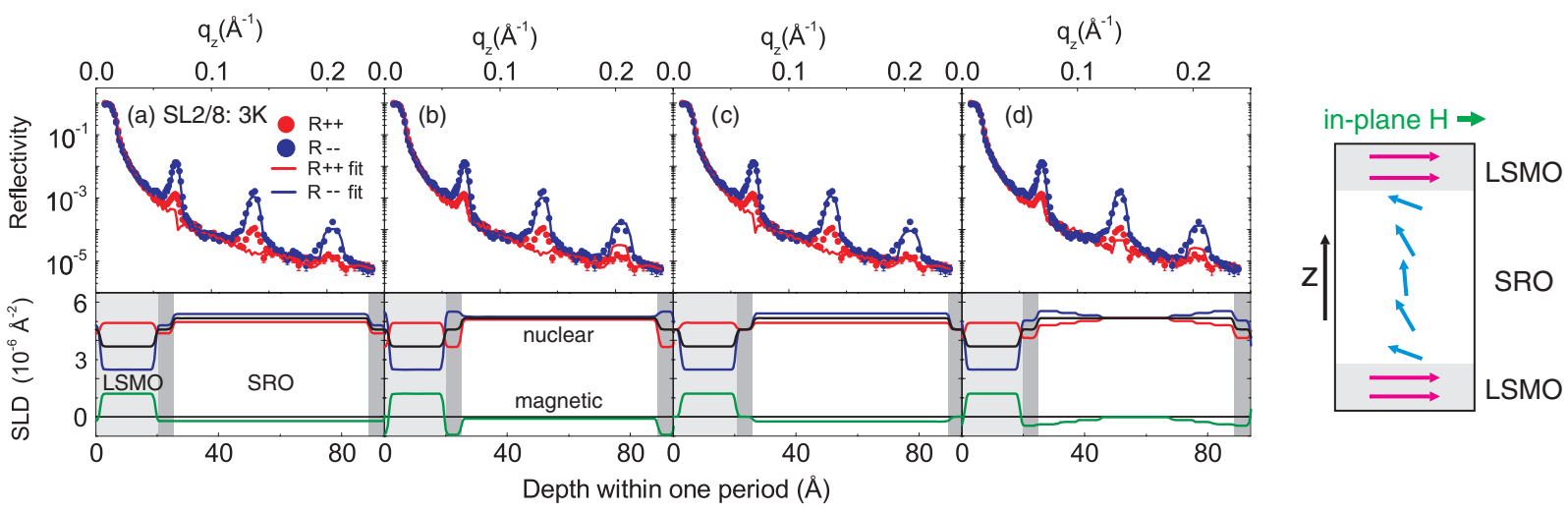

FIG. 5. (Color online) PNR results on SL2/8 at $3 \mathrm{~K}$. The symbols and colors were used in the same way as in Fig. 3. The magnetic depth profile at each temperature is given in the bottom row. The sketch shows a schematic of the spin configuration below $\mathrm{T}_{C}^{\mathrm{SRO}}$. 


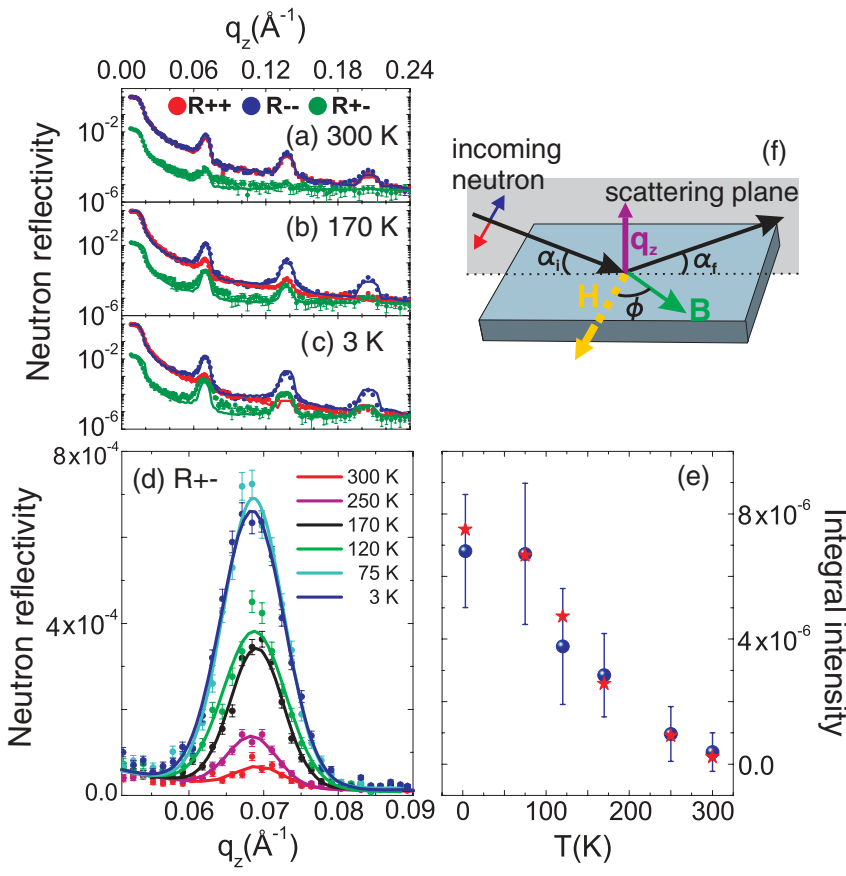

FIG. 6. (Color online) (a)-(c) Non-spin-flip and spin-flip spectra (symbols) and fits results (lines) on SL2/8. The symbols and colors were used in the same way as in Fig. 3. The polarization efficiency of $98.5 \%$ was considered for all fits. (d) Spin-flip data (symbol) and Gaussian fits (lines) at several temperatures at the first Bragg peaks. (e) The integral intensities of the spin-flip signal in (d) as a function of temperature. Red stars show the calculated values described in the text. (f) Diagram of the scattering geometry.

enhanced magnetization at the interface is thus required to fully describe the PNR data.

Analogous measurements and data analysis were performed on SL2/8. As shown in Figs. 3(c) and 3(d), the experimental PNR data and calculated results at 300 and $170 \mathrm{~K}$ show a similar behavior as those for the SL2/5 system. The total magnetic moments in the model calculations were constrained to $0.24 \mu_{B} / \mathrm{Mn}$ at $300 \mathrm{~K}, 2.21 \mu_{B} / \mathrm{Mn}$ at $170 \mathrm{~K}$, and $0.75 \mu_{B} / \mathrm{Mn}$ at $3 \mathrm{~K}$ [Fig. 1(b)]. To analyze the data at $3 \mathrm{~K}$, we tested similar magnetization profiles as for the SL2/5 system shown in Fig. 4. The three models with homogeneous SRO magnetization produce equally poor fits at the second-order SL peak, irrespective of the magnetization assumed in the intermixing layer [Figs. 5(a)-5(c)]. In contrast, this peak is significantly better fitted by a magnetization profile with an enhanced magnetization at the interface and a modulation in the SRO layer [Fig. 5(d)] comparable to SL2/5 [Fig. 4(d)].
Since the field of $0.1 \mathrm{~T}$ applied during the PNR measurements is not enough to saturate the LSMO and SRO magnetization, ${ }^{14}$ we have measured the spin-flip signals $R^{+-}$ and $R^{-+}$which are sensitive to the in-plane magnetic components perpendicular to the applied field. Figures 6(a)-6(c) show the PNR data up to the third SL Bragg peak. Upon cooling from room temperature, the intensity of the spin-flip signal at the first Bragg peak increases, and at $3 \mathrm{~K}$ it is comparable to $R^{++}$. The spin-flip intensity is proportional to $\left(\sum_{j} p_{j} \sin \phi_{j} \exp ^{i q_{z}(j-1) d}\right)^{2}$, where $p_{j}$ is the magnetic SLD of the $j$ th layer and $\phi_{j}$ is the angle between the direction of the magnetic field and the magnetic vector in the $j$ th layers. The results of fits with this model are presented as lines in the figure. Figures 6(d) and 6(e) present the spin-flip signal and its integrated intensity at the first Bragg peak as a function of temperature. The stars in Fig. 6(e) represent the calculated values obtained from the fits with $\phi=30^{\circ}$ in all layers. Despite its simplicity, this assumption leads to a good fit. This means that the in-plane magnetic moments of the $\mathrm{Mn}$ and Ru ions are $30^{\circ}$ canted from the field, and there is no modulation along the direction perpendicular to the field.

While PNR is only sensitive to the in-plane component of the magnetic moments, the combination of PNR and magnetometry data yields a comprehensive picture of the magnetization profile. We can thus attribute the enhanced outof-plane magnetization of both samples at low temperatures to the inside of the SRO layers, where the in-plane magnetization vanishes [Figs. 4(d) and 5(d)]. This implies a noncollinear magnetization profile as shown in the inset of Fig. 5. The origin of the noncollinearity is the competition between the antiferromagnetic Ru-Mn exchange coupling across the interface, which forces the $\mathrm{Ru}$ moments to coalign with the in-plane $\mathrm{Mn}$ at the interface, and the magnetocrystalline anisotropy of the $\mathrm{Ru}$ moments, which induces out-of-plane alignment away from the interfaces. The resulting spin configuration is similar to the "exchange spring" structures observed in multilayers composed of ferromagnetic elements or alloys with different coercivities. ${ }^{21-23}$ Since the magnetotransport properties of ferromagnetic SRO have recently commanded considerable attention, ${ }^{2-5}$ the ability to generate and manipulate such structures at epitaxial interfaces opens up interesting perspectives for spintronic device applications.

This work was supported by the Deutsche Forschungsgemeinschaft within the framework of the TRR80, project $\mathrm{C} 1$. We thank M. Dudek for performing the hard-X-ray reflectivity measurements and Olaf Soltwedel for helping with the PNR measurements at FRM-II.

\footnotetext{
*v.hinkov@fkf.mpg.de

†b.keimer@fkf.mpg.de

${ }^{1}$ For a review, see Y. Tokura and N. Nagaosa, Science 288, 462 (2000).

${ }^{2}$ J. S. Dodge, C. P. Weber, J. Corson, J. Orenstein, Z. Schlesinger, J. W. Reiner, and M. R. Beasley, Phys. Rev. Lett. 85, 4932 (2000).

${ }^{3}$ Z. Fang, N. Nagaosa, K. S. Takahashi, A. Asamitsu, R. Mathieu,

T. Ogasawara, H. Yamada, M. Kawasaki, Y. Tokura, and

K. Terakura, Science 302, 92 (2003).
}

${ }^{4}$ Y. Kats, I. Genish, L. Klein, J. W. Reiner, and M. R. Beasley, Phys. Rev. B 70, 180407(R) (2004).

${ }^{5}$ M.-H. Kim, G. Acbas, M.-H. Yang, M. Eginligil, P. Khalifah, I. Ohkubo, H. Christen, D. Mandrus, Z. Fang, and J. Cerne, Phys. Rev. B 81, 235218 (2010).

${ }^{6}$ J. Chakhalian, J. W. Freeland, G. Srajer, J. Strempfer, G. Khaliullin, J. C. Cezar, T. Charlton, R. Dalgliesh, C. Bernhard, G. Cristiani, H.-U. Habermeier, and B. Keimer, Nat. Phys. 2, 244 (2006). 
${ }^{7}$ J. Chakhalian, J. W. Freeland, H.-U. Habermeier, G. Cristiani, G. Khaliullin, M. van Veenendaal, and B. Keimer, Science 318, 1114 (2007).

${ }^{8}$ J. Hoppler, J. Stahn, Ch. Niedermayer, V. K. Malik, H. Bouyanfif, A. J. Drew, M. Rössle, A. Buzdin, G. Cristiani, H.-U. Habermeier, B. Keimer, and C. Bernhard, Nature Mater. 8, 315 (2009).

${ }^{9}$ P. Yu, J.-S. Lee, S. Okamoto, M. D. Rossell, M. Huijben, C.-H. Yang, Q. He, J. X. Zhang, S. Y. Yang, M. J. Lee, Q. M. Ramasse, R. Erni, Y.-H. Chu, D. A. Arena, C.-C. Kao, L. W. Martin, and R. Ramesh, Phys. Rev. Lett. 105, 027201 (2010).

${ }^{10}$ A. Y. Borisevich, H. J. Chang, M. Huijben, M. P. Oxley, S. Okamoto, M. K. Niranjan, J. D. Burton, E. Y. Tsymbal, Y. H. Chu, P. Yu, R. Ramesh, S. V. Kalinin, and S. J. Pennycook, Phys. Rev. Lett. 105, 087204 (2010).

${ }^{11}$ L. E. Hueso, J. M. Pruneda, V. Ferrari, G. Burnell, J. P. Valdés-Herrera, B. D. Simons, P. B. Littlewood, E. Artacho, A. Fert, and N. D. Mathur, Nature (London) 445, 410 (2006).

${ }^{12}$ Y. Moritomo, A. Asamitsu, and Y. Tokura, Phys. Rev. B 51, 16491 (1995).

${ }^{13}$ A. Kanbayasi, J. Phys. Soc. Jpn. 41, 1876 (1976).
${ }^{14}$ M. Ziese, I. Vrejoiu, E. Pippel, P. Esquinazi, D. Hesse, C. Etz, J. Henk, A. Ernst, I. V. Maznichenko, W. Hergert, and I. Mertig, Phys. Rev. Lett. 104, 167203 (2010).

${ }^{15}$ M. Ziese, I. Vrejoiu, and D. Hesse, Appl. Phys. Lett. 97, 052504 (2010).

${ }^{16}$ Y. Choi, Y. C. Tseng, D. Haskel, D. E. Brown, D. Danaher, and O. Chmaissem, Appl. Phys. Lett. 93, 192509 (2008).

${ }^{17}$ M. Björck and G. Andersson, J. Appl. Crystallogr. 40, 1174 (2007).

${ }^{18}$ R. Hillebrand, E. Pippel, D. Hesse, and I. Vrejoiu, Phys. Status Solidi A 208, 2144 (2011).

${ }^{19}$ J. Hoppler, J. Stahn, H. Bouyanfif, V. K. Malik, B. D. Patterson, P. R. Willmott, G. Cristiani, H.-U. Habermeier, and C. Bernhard, Phys. Rev. B 78, 134111 (2008).

${ }^{20}$ S. Mandal and R. C. Budhani, J. Magn. Magn. Mater. 320, 3323 (2008).

${ }^{21}$ B. Laenens, N. Planckaert, J. Demeter, M. Trekels, C. L'abbé, C. Strohm, R. Rüffer, K. Temst, A. Vantomme, and J. Meersschaut, Phys. Rev. B 82, 104421 (2010).

${ }^{22}$ R. Röhlsberger, H. Thomas, K. Schlage, E. Burkel, O. Leupold, and R. Rüffer, Phys. Rev. Lett. 89, 237201 (2002).

${ }^{23}$ K. V. O’Donovan, J. A. Borchers, C. F. Majkrzak, O. Hellwig, and E. E. Fullerton, Phys. Rev. Lett. 88, 067201 (2002). 